\title{
Tight Approximation of the Bit Error Rate for BICM(-ID) Assuming Imperfect CSI
}

\author{
Susanne Godtmann, Student Member, IEEE, I-Wei Lai, Student Member, IEEE, Gerd Ascheid, Member, IEEE, \\ Tzi-Dar Chiueh, Senior Member, IEEE, and Heinrich Meyr, Fellow, IEEE
}

\begin{abstract}
In this correspondence, we analytically derive the bit error rate (BER) for bit-interleaved coded modulation (BICM) under the assumption of a temporally correlated flat Rayleigh fading channel. We assume the channel state information (CSI) to be unknown at receiver side and consider a linear minimum mean-squared error (LMMSE) channel estimator that relies on periodically inserted pilot symbols. We give asymptotic results for both non-iterative BICM and BICM with iterative decoding (BICM-ID). Furthermore, we show that the interpretation of the channel estimation error as an SNR degradation holds true in these cases.
\end{abstract}

Index Terms-Bit-interleaved coded modulation (BICM), bitinterleaved coded modulation with iterative decoding (BICM-ID), temporal correlated fading, imperfect channel state information (CSI), LMMSE channel estimation.

\section{INTRODUCTION}

$\mathbf{I}$ T has been discovered by Zehavi in 1992 [1] that bitinterleaving rather then symbol interleaving considerably increases the diversity order of the system. This concept was later utilized in bit-interleaved coded modulation (BICM) and has been extensively elaborated on in the fundamental work by Caire et al. in [2]. Furthermore, Li and Ritcey [3] demonstrated the applicability of the turbo concept to BICM. This approach was labeled as bit-interleaved coded modulation with iterative decoding (BICM-ID) and makes use of soft extrinsic feedback from the soft-decoder to the soft-demapper.

Most of the literature on BICM(-ID) abstracts from channel estimation and assumes perfect channel state information (CSI) at receiver side. In the realistic case that the channel is unknown at receiver side, pilot symbols need to be inserted periodically into the data symbol stream in order to estimate the channel. This concept is also known as pilot symbol assisted modulation (PSAM) and was introduced in [4] and further elaborated in [5].

It has been shown in [2], [6] and e.g. [7] that for perfect CSI the asymptotic BER performance for BICM and BICM-ID respectively can be tightly approximated analytically. Results on the asymptotic BER behavior of systems with imperfect CSI have been published in e.g. [4], [8], [9]. However, in

Manuscript received July 10, 2007; revised December 17, 2007; accepted February 14, 2008. The associate editor coordinating the review of this letter and approving it for publication was M. Saquib.

S. Godtmann, G. Ascheid, and H. Meyr are with the Institute for Integrated Signal Processing Systems, RWTH Aachen University, Aachen, Germany (email: godtmann@iss.rwth-aachen.de).

I. Lai and T. Chiueh are with the Graduate Institute of Electronics Engineering, National Taiwan University, Taipei, Taiwan.

S. Godtmann thanks the Deutsche Telekom Stiftung for its financial support.

Digital Object Identifier 10.1109/T-WC.2008.070752 these works uncoded transmission is treated. Later on, Huang and Ritcey [10] investigated the effects of imperfect CSI on the coded BER of BICM-ID. Huang and Ritcey follow the approach proposed in [9] and succeed in parameterizing the incomplete CSI in a variable $\rho$, which is a function of the channel dynamics, the channel estimator, and the signal-tonoise ratio (SNR). However, due to the complicated form of the joint probability density function (pdf) of the channel and its estimate one has to resort to (rather complicated) Monte Carlo simulation in order to evaluate one expression (Eq. (11) in [10]). Therefore, this approach provides only limited intuitive insights into the effects of the channel dynamics.

In this correspondence, we calculate an approximation for the coded BER for BICM(-ID) under the assumption of a correlated Rayleigh fading channel with imperfect CSI at receiver side. We consider an LMMSE channel estimator, that relies on periodically inserted pilot symbols (PSAM). We are able to obtain a tight approximation of the BER without making use of Monte Carlo simulation. The mathematical expression for the BER has then the same form as that of the BER for the case with perfect CSI. Comparing these results, we can introduce a so-called estimation loss with respect to a system with perfect CSI. The estimation loss is a function of the channel statistics, the SNR, and the pilot spacing.

We base our derivations on the fundamental work [2], where both the union bound and the so-called expurgated bound for the coded BER of BICM are deduced. We here concentrate on the expurgated bound that differs from the union bound in the sense that the former one only considers the most likely error event, i.e. nearest neighbor, whereas the latter one incorporates all possible error events [2]. However, it should be stressed that the fact that the so-called expurgated bound is an actual upper bound on the BER was only proved in [2] for the important case of square QAM signal sets with Gray labeling. Otherwise, it is only a tight approximation of the actual BER ${ }^{1}$. Since for the asymptotic case of BICM-ID the decision in the signal space reduces to a binary decision regardless of the modulation alphabet, the expurgated bound provides the same result as the union bound and is therefore an upper bound. However, as we need to make the (best-case) assumption of error free feedback (EFF) to evaluate the BER performance for BICMID, we do not get a BER bound for this case either, but a tight approximation. This issue does not arise from our specific

\footnotetext{
${ }^{1}$ In fact, it has been later disclosed by Sethuraman and Hajek [11] that the proof that the expurgated bound is an upper BER bound for QAM signal sets and Gray labeling is flawed. However, the results still represent a very tight approximation on the coded BER.
} 
approach, but is equally inherent in the other work published on this topic, e.g. [7], [10], [12].

\section{TRAnSmission Model}

The system model considered in this paper is shown in Fig. 1. Information bits are grouped into packets of $N$ bits, encoded with a convolutional code of rate $r=k_{c} / n$, interleaved and mapped onto an $M$-ary signal constellation set $\chi$ according to the mapping rule $\mu$. Then, pilot symbols are multiplexed into the data symbol stream. The spacing between the pilot symbols is denoted as $P_{s}$. The resulting data symbols are then transmitted over a flat Rayleigh fading channel that also suffers from additive white Gaussian noise (AWGN). The fading is assumed to be temporally correlated. The maximum Doppler spread normalized to the symbol duration is given by $F_{d}=f_{d} T_{s}$.

Under the assumption of perfect timing synchronization, the received baseband signal after matched filtering and sampling can be modelled as

$$
y_{k}=h_{k} \cdot x_{k}+n_{k},
$$

where $h_{k}$ is the complex fading coefficient at sampling instant $k$. The transmitted and the received symbol are denoted as $x_{k}$ and $y_{k}$, respectively. $n_{k}$ denotes samples of complexvalued AWGN with independent real and imaginary parts each having zero-mean and variance $\sigma_{n}^{2} / 2$. The total noise variance is given to $N_{0}=\sigma_{n}^{2}$. Furthermore, $\mathrm{E}\left\{\left|x_{k}\right|^{2}\right\}$ and $\mathrm{E}\left\{\left|h_{k}\right|^{2}\right\}$ are denoted as $\sigma_{x}^{2}$ and $\sigma_{h}^{2}$, respectively.

At receiver side, data symbols and pilot symbols are demultiplexed and the latter ones are used for linear minimum mean squared error (LMMSE) channel estimation [13], also called Wiener filtering. The filter length is given by $F_{W}$.

After channel estimation and equalization, the received symbols $y_{k}$ are soft-demapped and the calculated bit metrics are deinterleaved and passed on to the decoder. Thereafter, the decoder either outputs the decoded bits directly (noniterative BICM) or calculates extrinsic information on the coded data bits and feedbacks these metrics back to the softdemapper (BICM-ID). For a detailed explanation of the softdemapper and the extrinsic information exchange, please refer to e.g. [7], [12].

For later use, we make the following definition: Let $\chi_{b}^{i}$ denote the subset of $\chi$ whose labels have the binary value $b$ at the $i$-th bit position, with $i \in[1, I]$, and $I=\log _{2}(M)$.

\section{ASYMPTOTIC BER FOR BICM(-ID) WITH PERFECT CSI}

As already stated in the introduction, we base our derivations on the expurgated bound for the coded BER of BICM. In this section, we will shortly summarize the derivations in [2]. All derivations in this section and the subsequent section are based on the assumption of infinite block length and, therefore, infinite interleaver length.

The BER can be bounded by:

$$
P_{b} \leq \frac{1}{k_{c}} \sum_{d=d_{\min }}^{\infty} W_{I}(d) f\left(d, \mu, \chi, E_{s} / N_{0}\right),
$$

where $W_{I}(d)$ is the total input weight of error events at Hamming distance $d$. The minimum Hamming distance is denoted as $d_{\min }$. The pairwise error probability (PEP) $f\left(d, \mu, \chi, E_{s} / N_{0}\right)$ depends on the Hamming distance, the mapping $\mu$, the signal constellation $\chi$ and the SNR. It can be approximated by the expurgated PEP $f_{\mathrm{ex}}\left(d, \mu, \chi, E_{s} / N_{0}\right)[2],[11]$ :

$$
\begin{aligned}
f\left(d, \mu, \chi, E_{s} / N_{0}\right) & \approx f_{\mathrm{ex}}\left(d, \mu, \chi, E_{s} / N_{0}\right) \\
& =\frac{1}{2 \pi j} \int_{c-j \infty}^{c+j \infty}\left[\psi_{\mathrm{ex}}(s)\right]^{d} \frac{d s}{s},
\end{aligned}
$$

where $c$ is chosen such that the integral converges [2]. $\psi_{\mathrm{ex}}(s)$ is given as

$$
\psi_{\mathrm{ex}}(s)=\frac{1}{I 2^{I}} \sum_{i=1}^{I} \sum_{b=0}^{1} \sum_{x \in \chi_{b}^{i}} \Phi_{\Delta(x, z)}(s),
$$

where $\Phi_{\Delta(x, z)}(s)$ is the bilateral Laplace transform of the probability density function (pdf) of the metric difference $\Delta(x, z) . x$ is the correct symbol, whereas $z$ represents the symbol that is erroneously detected due to an error event. $\Delta(x, z)$ is formally defined to be [2]:

$$
\begin{aligned}
\Delta(x, z) & =\log p(y \mid x)-\log p(y \mid z) \\
\text { with } p(y \mid x) & =\frac{1}{\pi N_{0}} \exp \left\{-\frac{|y-h x|^{2}}{N_{0}}\right\}
\end{aligned}
$$

Since an error occurs if $\Delta(x, z)<0$, we are only interested in the probability $P(\Delta(x, z)<0)$ (Eq. (47) in [2]). Therefore, the constant factor $1 / N_{0}$ is dropped:

$$
\Delta(x, z)=|y-h z|^{2}-|y-h x|^{2} .
$$

The metric difference $\Delta(x, z)$ differs for (non-iterative) BICM and BICM-ID. In order to avoid confusion, we introduce the following notation:

$$
\Delta(x, z):= \begin{cases}\Delta(x, \tilde{z})_{P} & \text { for BICM } \\ \Delta(x, \hat{z})_{P} & \text { for BICM-ID. }\end{cases}
$$

The subscript $(\cdot)_{P}$ stands for "perfect CSI". In the later evaluation, when imperfect CSI is introduced, it is replaced by $(\cdot)_{I}$.

In the case of the expurgated bound for (non-iterative) $\mathrm{BICM}, \tilde{z}=\tilde{z}(x) \in \chi_{\bar{b}}^{i}$ is defined to be the nearest constellation point of $x \in \chi_{b}^{i}$ for which $\tilde{z} \in \chi_{\bar{b}}^{i}$ holds, where $\bar{b}$ indicates the negation of $b$. When the asymptotic BER behavior of BICMID is considered, the assumption of EFF is made [7], [12]. Irrespective of the signal constellation and the mapping, the decision on signal constellation level then reduces to a binary decision. Therefore, given $x \in \chi_{b}^{i}$, the set $\chi_{\bar{b}}^{i}$ then only contains one term $\hat{z}=\hat{z}(x)$, whose labels have the same binary bit values as those of $x$ except at the $i$-th bit position [12].

According to Eq. (23) in [7], the bilateral Laplace transform can be calculated as

$$
\begin{aligned}
\Phi_{\Delta(x, z)_{P}}(s) & =\mathrm{E}_{|h|}\left\{\mathrm{E}\left\{e^{-s \Delta(x, z)_{P} \mid}|h|\right\}\right\} \\
& =\frac{1}{1-\sigma_{h}^{2}|d|^{2} s\left(N_{0} s-1\right)},
\end{aligned}
$$

with $d=x-\tilde{z}$ for BICM and $d=x-\hat{z}$ for BICM-ID. 


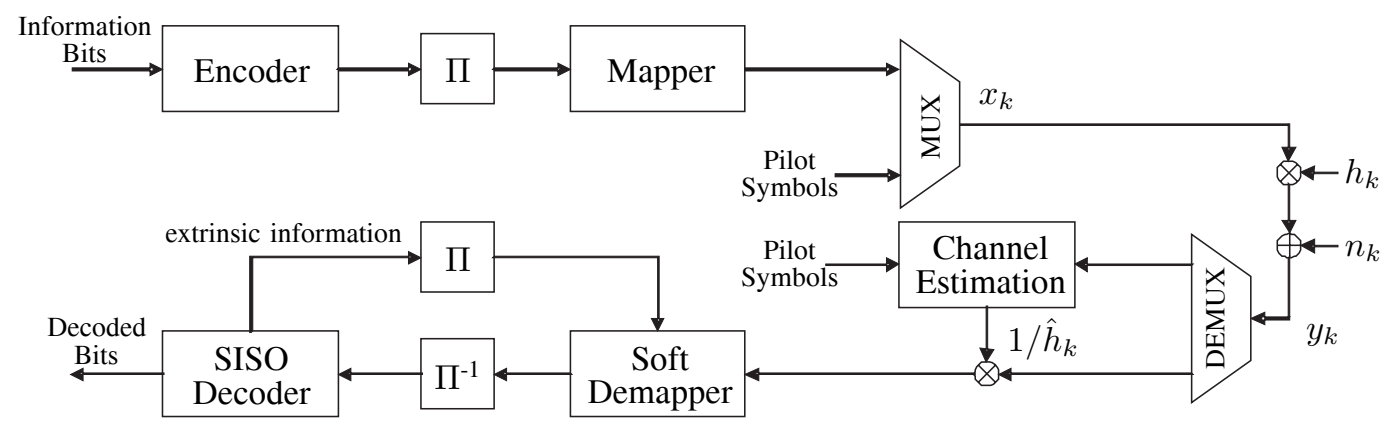

Fig. 1. System Model

In order to use normalized quantities, we normalize the distance $d$ to $\sigma_{x}^{2}$ :

$$
\Phi_{\Delta(x, z)_{P}}(s)=\frac{1}{1-\sigma_{h}^{2} \sigma_{x}^{2}|\tilde{d}|^{2} s\left(N_{0} s-1\right)} .
$$

With the means of Gauss-Chebyshev quadrature [14], it is then possible to numerically compute the PEP given in (3) and resolve (2). The result gives an approximation of the BER for a BICM(-ID) system.

\section{AsYMPTOTIC BER FOR BICM(-ID) WITH IMPERFECT CSI}

In this section, we calculate the bilateral Laplace transform of $\Phi_{\Delta(x, \tilde{z})}(s)$ (BICM) and $\Phi_{\Delta(x, \hat{z})}(s)$ (BICM-ID) under the assumption of imperfect CSI. As there is no fundamental difference in the derivation, we will here only show the calculations for (non-iterative) BICM.

In order to determine the bilateral Laplace transform $\Phi_{\Delta(x, \tilde{z})_{I}}(s)$, the metric difference $\Delta(x, \tilde{z})_{I}$ needs to be calculated for the case of imperfect CSI. We make the assumption that the receiver exhibits LMMSE channel estimation, such that $\mathrm{E}\left\{\hat{h} e^{*}\right\}=0$ holds true, where $(\cdot)^{*}$ denotes the complex conjugate. $e$ is the estimation error $e=\hat{h}-h$. In accordance to (6), the metric difference is given to

$$
\Delta(x, \tilde{z})_{I}=|y-\hat{h} \tilde{z}|^{2}-|y-\hat{h} x|^{2} .
$$

Introducing $d=x-\tilde{z}$, replacing $y$ by $y=h x+n$ and making use of $e=\hat{h}-h$, yields

$$
\begin{aligned}
\Delta(x, \tilde{z})_{I}= & 2 \operatorname{Re}\left\{n \hat{h}^{*} d^{*}\right\}+2|\hat{h}|^{2} \operatorname{Re}\left\{x d^{*}\right\} \\
& -2 \operatorname{Re}\left\{e x \hat{h}^{*} d^{*}\right\}-|\hat{h}|^{2}\left(|x|^{2}-|\tilde{z}|^{2}\right) \\
= & 2 \operatorname{Re}\left\{n \hat{h}^{*} d^{*}\right\}+|\hat{h}|^{2}\left(|x|^{2}-2 \operatorname{Re}\left\{x \tilde{z}^{*}\right\}+|\tilde{z}|^{2}\right) \\
& -2 \operatorname{Re}\left\{e x \hat{h}^{*} d^{*}\right\} \\
= & 2 \operatorname{Re}\left\{n \hat{h}^{*} d^{*}\right\}+|\hat{h} d|^{2}-2 \operatorname{Re}\left\{e x \hat{h}^{*} d^{*}\right\} \cdot(11)
\end{aligned}
$$

As $e$ and $n$ are independent complex Gaussian random variables, $\Delta(x, \tilde{z})_{I}$ is also a Gaussian random variable if $\hat{h}$ is given. Its mean value and its variance are

$$
\begin{aligned}
\mu_{\Delta} & =|\hat{h} d|^{2}, \\
\sigma_{\Delta}^{2} & =2|\hat{h} d|^{2}\left(|x|^{2} \sigma_{e}^{2}+N_{0}\right) .
\end{aligned}
$$

It should be stressed that (13) only holds valid, if $\sigma_{e}^{2}$ is independent of the time index $k$. It is shown in [15] that this is the case when the channel is sampled at least with Nyquist rate.
In accordance with (8), we obtain the conditional bilateral Laplace transform of the pdf of $\Delta(x, \tilde{z})_{I}$ as

$$
\Phi_{\Delta(x, \tilde{z})_{I}}(s)=\mathrm{E}_{|\hat{h}|}\left\{\mathrm{E}\left\{\exp \left(-\mu_{\Delta} s+\frac{1}{2} \sigma_{\Delta}^{2} s^{2}\right)|| \hat{h} \mid\right\}\right\} .
$$

In the next step, we insert the results obtained in (12) and (13) and then get

$$
\begin{aligned}
& \Phi_{\Delta(x, \tilde{z})_{I}}(s) \\
& \quad=\mathrm{E}_{|\hat{h}|}\left\{\mathrm{E}\left\{\exp \left(|\hat{h}|^{2}|d|^{2}\left(\left(N_{0}+\sigma_{e}^{2}|x|^{2}\right) s^{2}-s\right)\right)|| \hat{h} \mid\right\}\right\} .
\end{aligned}
$$

As $|\hat{h}|$ is a Rayleigh distributed random variable, we can use

$$
\mathrm{E}_{q}\left\{\mathrm{E}\left\{e^{A q^{2}} \mid q\right\}\right\}=\frac{1}{1-2 \sigma_{q}^{2} A} \text { if } q \sim \operatorname{Rayleigh}\left(\sigma_{q}^{2}\right)
$$

in order to simplify (15) to

$$
\Phi_{\Delta(x, \tilde{z})_{I}}(s)=\frac{1}{1-\left(\sigma_{h}^{2}-\sigma_{e}^{2}\right) \sigma_{x}^{2}|\tilde{d}|^{2} s\left[\left(N_{0}+\sigma_{e}^{2}|x|^{2}\right) s-1\right]},
$$

where $\tilde{d}$ is corresponds to $d$ normalized to $\sigma_{x}^{2}$. Note that $\Phi_{\Delta(x, \hat{z})_{I}}(s)$ for BICM-ID looks similar as (17), except that $d$ is then defined to be $d:=x-\hat{z}$.

As already described in the last paragraph of Section III, we then make use of Gauss-Chebyshev quadrature in order to compute the PEP and then the BER.

\section{RELATion BETwEen CHANNEl Estimation ERroR AND SNR DEGRADATION}

In this section, we analytically show that the channel estimation error can be seen as an additional SNR degradation. This interpretation has already been successfully applied in [15] in the context of the achievable rate and in [8] in the context of uncoded BER.

Taking the ratio of the signal and the noise components 
of (17) yields:

$$
\begin{aligned}
\gamma_{I} & =\frac{\sigma_{x}^{2}\left(\sigma_{h}^{2}-\sigma_{e}^{2}\right)}{N_{0}+\sigma_{e}^{2}|x|^{2}} \\
& =\frac{\sigma_{h}^{2} \sigma_{x}^{2}}{N_{0}} \cdot \frac{1-\frac{\sigma_{e}^{2}}{\sigma_{h}^{2}}}{1+\frac{\sigma_{e}^{2}}{\sigma_{h}^{2}} \cdot \frac{|x|^{2} \sigma_{h}^{2}}{N_{0}}} \\
& =\underbrace{\frac{\sigma_{h}^{2} \sigma_{x}^{2}}{N_{0}}}_{\gamma_{P}} \cdot \underbrace{\frac{1-\sigma_{e}^{2} / \sigma_{h}^{2}}{1+\sigma_{e}^{2} / \sigma_{h}^{2} \cdot E_{s} / N_{0}}}_{L_{\mathrm{Est}}},
\end{aligned}
$$

where the first factor $\gamma_{P}$ clearly is the ratio of the signal and the noise components of (9) for the perfect CSI case and the second factor denotes the loss due to imperfect channel estimation. It should be stressed that the comparison in (18) is made assuming the same $\sigma_{x}^{2}$ in (9) and in (17).

However, in order to make an adequate comparison, we assume a constant power budget at the transmitter. The code rate and the size of the modulation alphabet are considered to be the same for both cases. Therefore, we have to consider the insertion of pilot symbols as an increase in the number of transmit symbols and a decrease in the $E_{s} / N_{0}$ of the channel.

$$
E_{s} / N_{0}=\left(E_{s} / N_{0}\right)_{P} \cdot \frac{P_{s}-1}{P_{s}},
$$

where $\left(E_{s} / N_{0}\right)_{P}$ is the $E_{s} / N_{0}$ for a system that does not make use of pilot symbols. Note that a completely fair comparison would also require a constant number of transmit symbols. However, in order to realize this, the code rate and the modulation alphabet size need to be adjusted depending on $P_{s}$. As this entails a large scope of parameters that need to be optimized in order to guarantee comparability, we here chose to restrict only to the "constant power budget condition".

Therefore, as an SNR degradation factor with respect to a system with perfect CSI and no pilot symbols, we get:

$$
L_{\mathrm{SNR}}=\left(\frac{P_{s}-1}{P_{s}}\right) \cdot \frac{1-\sigma_{e}^{2} / \sigma_{h}^{2}}{1+\sigma_{e}^{2} / \sigma_{h}^{2} \cdot\left(E_{s} / N_{0}\right)_{P} \cdot\left(\frac{P_{s}-1}{P_{s}}\right)},
$$

where the first factor is due to the energy loss by inserting pilot symbols, whereas the second factor represents the estimation loss. Obviously, the second term approaches one for $\sigma_{e}^{2} \rightarrow 0$.

Note that for the LMMSE estimator, $\sigma_{e}^{2}$ can be calculated analytically. For a rectangular fading spectrum and $F_{W} \rightarrow \infty$ it can be given as (cf. Eq. (25) in [15]):

$$
\sigma_{e}^{2}=\frac{2 F_{d} P_{s}}{2 F_{d} P_{s}+\left(E_{s} / N_{0}\right)_{P} \cdot \frac{P_{s}-1}{P_{s}}} \cdot \sigma_{h}^{2} .
$$

It is then possible to insert (21) into (20) and to analytically find the $P_{s}$ that minimizes (20).

The SNR loss due to pilot symbol insertion and imperfect channel estimation is depicted in Fig. 2 for different channel dynamics. The channel estimation error in (20) is calculated according to (21).

\section{Simulation Results}

In this section, we demonstrate that the numerical simulation results match the calculated asymptotic BER results. Fig. 3 depicts the analytical asymptotic BER (lines

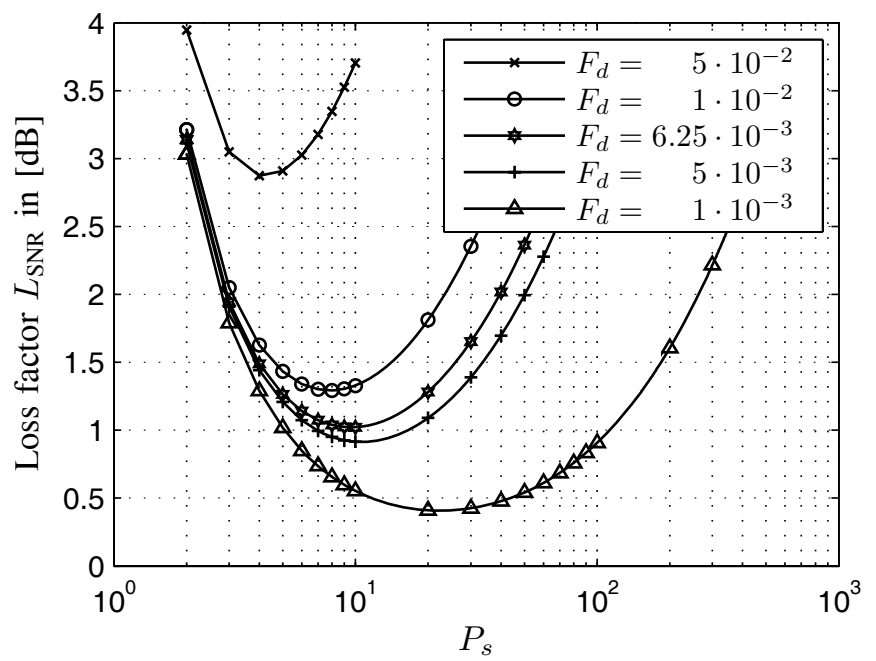

Fig. 2. Loss factor $L_{\mathrm{SNR}}$ vs. $P_{s} ;\left(E_{s} / N_{0}\right)_{P}=10 \mathrm{~dB}$.

without markers) and the BER obtained through Monte Carlo simulation (lines with markers). "P-CSI" denotes the case of perfect channel state information, whereas "I-CSI" denotes the case of imperfect channel state information. For the latter case (in accordance with [10]), we choose the pilot spacing to $P_{s}=51$ and the length of the LMMSE channel estimator to $F_{W}=10$. (The results are equally accurate for other $F_{W}$.) The correlation properties of the fading process are determined by the Jakes' spectrum and the normalized maximum Doppler shift is set to $F_{d}=6.25 \cdot 10^{-3}$. In order to account for the fact that for (non-iterative) BICM and BICM-ID different BER operating points occur, we choose different generator polynomials for the two cases. For the Soft-Input-Soft-Output-Decoder, we apply the BCJR algorithm with its typical max-log MAP simplification. As the analytical approximations are for $N \rightarrow \infty$, we choose a block length that is rather large, $N=6 \cdot 10^{4}$. Furthermore, the analytical results for BICM-ID rely on the assumption of EFF. Therefore, we choose a sufficiently high number of iterations.

For (non-iterative) BICM (Fig. 3(a)) and BICM-ID (Fig. 3(b)), we note that the simulated BER tightly approaches the analytical asymptotic BER for high SNR. The gap between the analytical expression and the simulated BER vanishes for high SNR due to the fact that for the derivation of the analytical expression only nearest neighbor error events are considered.

The possibility to analytically determine a tight approximation of the coded BER for BICM(-ID) with imperfect channel state information significantly facilitates the dimensioning of both the pilot spacing $P_{s}$ and the filter length $F_{W}$ for a flat Rayleigh fading channel. It is now feasible to determine the optimum $P_{s}$ and $F_{W}$ for a specified operating point without running extensive Monte Carlo simulations.

Exemplarily, we show the BER vs. the pilot spacing $P_{s}$ in Fig. 4 for the filter length $F_{W}=10$ (straight line, diamond markers) and for $F_{W} \rightarrow \infty$ (straight line, circle markers). The performance gap between these curves is the so-called implementation loss of the Wiener filter.

In order to abstract from a data-aided channel estimation 


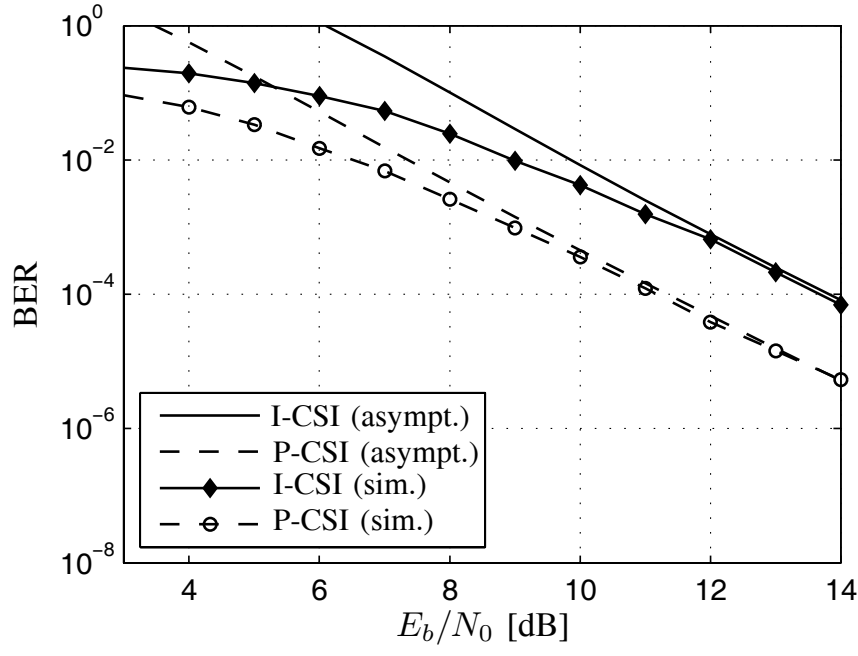

(a) BICM, Gray mapping, Conv. Code $\{5,7\}_{8}$

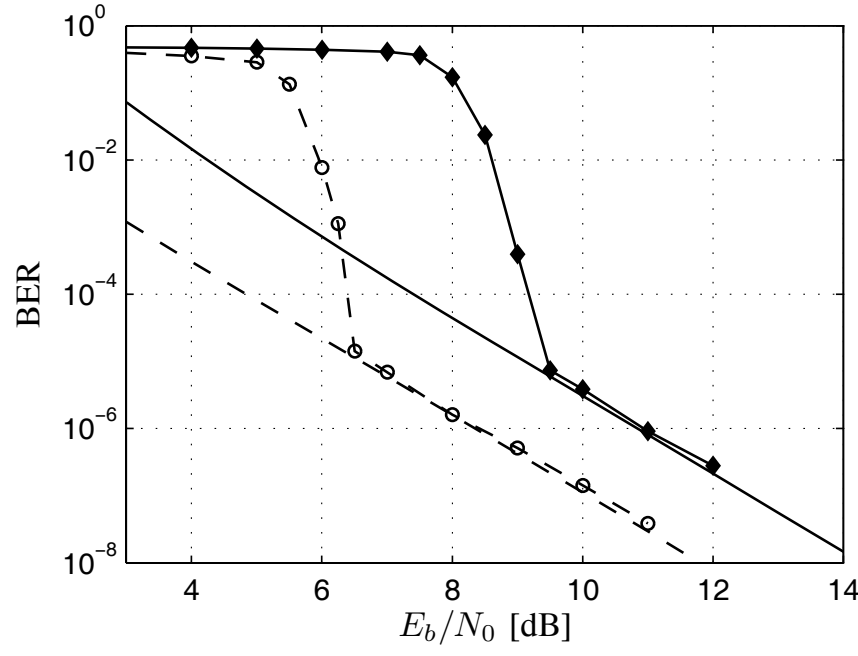

(b) BICM-ID, 10 iterations, SSP mapping [12], Conv. Code $\{15,17\}_{8}$

Fig. 3. BER vs. $E_{b} / N_{0} ; N=6 \cdot 10^{4}, 8$-PSK, $r=1 / 2, f_{d} T_{s}=6.25 \cdot 10^{-3}, P_{s}=51, F_{W}=10$.

unit (PSAM), we also show the BER performance approximations for iterative channel estimation (e.g. [16]), i.e. where the channel estimation unit gets feedback from the SISO decoder (dashed curves). Here, we assume error free feedback (EFF) from the SISO decoder to the channel estimation unit. This implies that the Wiener filter uses channel observations at all time instances for channel estimation. The variance of the channel estimation error $\sigma_{e}^{2}$ in the expression of the loss factor (20) must then be computed for the channel dynamic $F_{d}$, for the pilot spacing $P_{s}=1$ and for the SNR $E_{s} / N_{0}=\left(E_{s} / N_{0}\right)_{P}\left(P_{s}-1\right) / P_{s}$. The solid curve with triangular markers belongs to an (artificial) system with pilot symbols but perfect CSI for the data symbol positions. The difference to the lower dashed curve almost vanishes for $P_{s} \rightarrow \infty$. However, caution is necessary in drawing conclusions from the dashed curves. They do not reveal any information whether the iterative channel estimation algorithms converges. Above a certain pilot spacing, this is no longer the case. The determination of this threshold is subject to ongoing research.

The undermost horizontal line (without markers) corresponds to the case of perfect CSI without pilot symbols. Note that the two curves for perfect CSI are independent of the channel dynamics and that the difference between the BER for perfect CSI and for imperfect CSI is large, even for small channel dynamics as used in this example. This demonstrates that for the case of non-negligible channel dynamics, the evaluation of system performance based on perfect CSI is of limited practical value.

From Fig. 4, we also see that $P_{s}=51$ that is chosen for the evaluations in Fig. 3(b) is suboptimum in terms of the BER. In order to minimize the BER for the given channel correlation properties and LMMSE data-aided channel estimation, the $P_{s}$ should be chosen to $P_{s} \approx 10$. However, the advantage of a high pilot spacing is of course a higher data rate. Therefore, we here clearly see the tradeoff between BER and data rate.

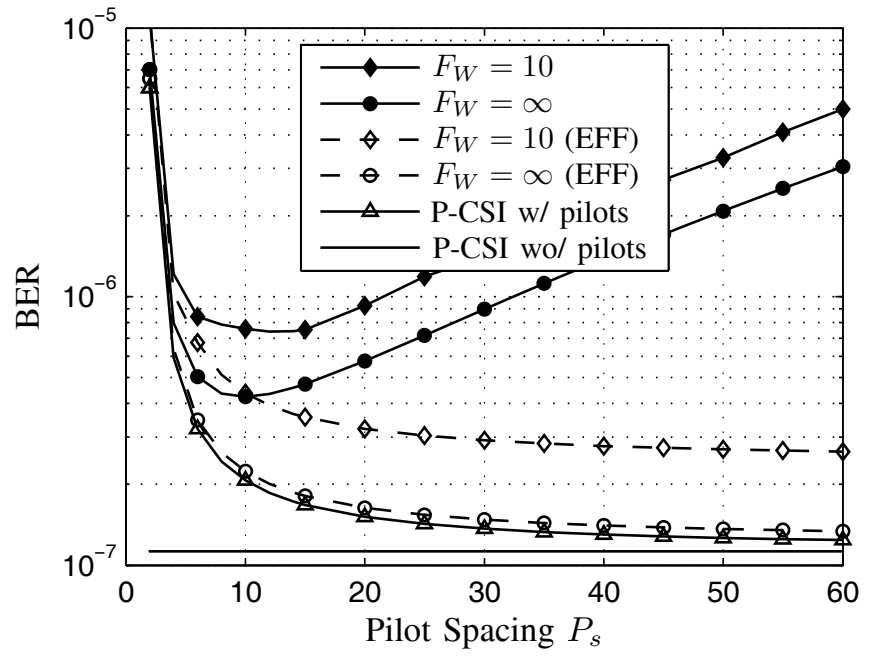

Fig. 4. Asymptotic BER vs. $P_{s}$ for BICM-ID; $E_{b} / N_{0}=10 \mathrm{~dB}$,

8-PSK, SSP mapping, Conv. Code $\{15,17\}_{8}, r=1 / 2, f_{d} T_{s}=6.25 \cdot 10^{-3}$.

\section{CONCLUSION}

In this correspondence, we investigate the asymptotic BER performance of BICM(-ID) under the assumption of imperfect channel state information. We provide analytical expressions for the BER under the assumption of an LMMSE channel estimator that is optimum for pilot symbol assisted modulation. Furthermore, we show by means of Monte Carlo simulation that the obtained results tightly approximate the asymptotic BER behavior of a BICM(-ID) system. The analytical BER expressions are therefore valuable for practical system design.

Additionally, we validate the interpretation of the residual channel estimation error as SNR degradation.

\section{REFERENCES}

[1] E. Zehavi, "8-PSK Trellis codes for a Rayleigh channel," IEEE Trans. Commun., vol. 40, no. 5, pp. 873-884, May 1992.

[2] G. Caire, G. Taricco, and E. Biglieri, "Bit-interleaved coded modulation," IEEE Trans. Inform. Theory, vol. 44, no. 3, pp. 927-946, May 1998. 
[3] X. Li and J. A. Ritcey, "Bit-interleaved coded modulation with iterative decoding using soft feedback," IEEE Electron. Lett., vol. 34, no. 10, pp. 942-943, May 1998.

[4] J. K. Cavers, "An analysis of pilot symbol assisted modulation for Rayleigh fading channels," IEEE Trans. Veh. Technol., vol. 40, no. 4, pp. 686-693, Nov. 1991.

[5] H. Meyr, M. Moeneclaey, and S. Fechtel, Digital Communication Receivers: Synchronization, Channel Estimation and Signal Processing, 1st ed. New York: John Wiley \& Sons, 1998.

[6] A. Martinez, A. Guillén i Fàbregas, and G. Caire, "Error probability analysis of bit-interleaved coded modulation," IEEE Trans. Inform. Theory, vol. 52, no. 1, pp. 262-271, Jan. 2006.

[7] A. Chindapol and J. A. Ritcey, "Design, analysis, and performance evaluation for BICM-ID with square QAM constellations in Rayleigh fading channels," IEEE J. Select. Areas Commun., vol. 19, no. 5, pp. 944-957, May 2001.

[8] A. Aghamohammadi and H. Meyr, "On the error probability of linearly modulated signals on Rayleigh frequency-flat fading channels," IEEE Trans. Commun., vol. 38, no. 11, pp. 1966-1970, Nov. 1990.

[9] X. Tang, M.-S. Alouini, and A. J. Goldsmith, "Effect of channel estimation error on M-QAM BER performance in Rayleigh fading," IEEE Trans. Commun., vol. 47, no. 12, pp. 1856-1864, Dec. 1999.
[10] Y. Huang and J. A. Ritcey, "16-QAM BICM-ID in fading channels with imperfect channel state information," IEEE Trans. Wireless Commun., vol. 2, no. 5, pp. 1000-1007, Sept. 2003.

[11] V. Sethuraman and B. Hajek, "Comments on "bit-interleaved coded modulation"," IEEE Trans. Inform. Theory, vol. 52, no. 4, pp. 1795-1797, Apr. 2006.

[12] X. Li, A. Chindapol, and J. A. Ritcey, "Bit-interleaved coded modulation with iterative decoding and 8PSK signaling," IEEE Trans. Commun., vol. 50, no. 8, pp. 1250-1257, Aug. 2002.

[13] S. M. Kay, Fundamentals of Statistical Signal Processing, vol. I: Estimation Theory. Prentice Hall PTR, 1993.

[14] E. Biglieri, G. Caire, G. Taricco, and J. Ventura-Traveset, "Simple method for evaluating error probabilities," IEE Electron. Lett., vol. 32, no. 3, pp. 191-192, 1996.

[15] J. Baltersee, G. Fock, and H. Meyr, "An information theoretic foundation of synchronized detection," IEEE Trans. Commun., vol. 49, no. 12, pp. 2115-2123, Dec. 2001.

[16] M. C. Valenti and B. D. Woerner, "Iterative channel estimation and decoding of pilot symbol assisted Turbo codes over flat-fading channels," IEEE J. Select. Areas Commun., vol. 19, no. 9, pp. 1697-1705, Sept. 2001. 